\title{
VEGETATION STRUCTURE AND THREATS TO MONTANE TEMPERATE ECOSYSTEMS IN HINDUKUSH RANGE, SWAT, PAKISTAN
}

\author{
Ali, A. ${ }^{1,2^{*}}-$ BADSHAH, L. ${ }^{2}-$ HUSSAIN, F. $^{3}$ \\ ${ }^{1}$ Govt. AKL Post Graduate College Matta, Swat, Pakistan \\ (phone: +92-333-948-5390) \\ ${ }^{2}$ Department of Botany, University of Peshawar, Pakistan \\ (phone: +92-333-894-4128) \\ ${ }^{3}$ Department of Microbiology, Sarhad University of Science and Information Technology \\ Peshawar, Pakistan \\ (phone: +92-333-933-6777) \\ *Corresponding author \\ e-mail: asghartk@gmail.com \\ (Received $14^{\text {th }}$ May 2018; accepted $5^{\text {th }}$ Jul 2018)
}

\begin{abstract}
This phytosociological study conducted during 2013-2017 summed up 15 communities including 5 each for trees, shrubs and herbs. Quantitative attributes of vegetation were recorded by using quadrate method i.e. $10 \mathrm{~m}^{2}$ trees, $5 \mathrm{~m}^{2}$ for shrubs and $1 \mathrm{~m}^{2}$ quadrate for herbs. Edaphic attributes were enumerated by employing standard methods. Among tree species highest Family Importance Value was recorded for Pinnaceae (190.85) and lowest for Anacardiaceae (2.39). For shrubs Rosaceae (101.44) had highest FIV and lowest for Rubiaceae (1.89). For herbaceous members highest FIV was recorded for Poaceae (182.15) and lowest for Paeoniaceae (0.42). Three communities viz. Pinus- Diospyros- Quercus community, Indigofera- Cotoneaster- Jasminum community and Apluda-Cynodon-Heteropogon community were established at Lower Chail site. While Upper Chail comprised Pinus-Quercus-Juglans, Rubus-Isodon-Ricinus and Apluda-Heteropogon-Poa communities. Community structure was quite different at Shanku site which established Quercus-Abies-Pinus, Sarcococca-Daphne-Berberis and Cynodon-Apluda-Bergenia communities respectively. Similarly, Cedrus-Abies-Pinus, ParrotiopsisVibernum-Berberis and Chrysopogon-Themeda-Cynodon communities were established at Dabargai. At Bishigram, Picea-Pinus-Abies, Parrotiopsis-Cotoneaster-Indigofera and Cenchrus-Medicago-Rumex communities were present. Soils were mildly acidic and had low organic matter content. Vegetation was found to be under severe biotic stress. Deforestation is threatening these ecosystems and conservation of montane temperate forests in this zone is need of the day.
\end{abstract}

Keywords: phytosociology, edaphic parameters, maturity index, similarity index, moist temperate vegetation

\section{Introduction}

Study of plant communities and their classification is termed as phytosociology (Mishra et al., 2012; Ahmad and Shaukat, 2012). Primarily phytosociology helps in understanding the multilateral relationships between plants and their environment. It deals with quantitative, qualitative and synthetic attributes of plant communities (Badshah et al., 2016; Ali et al., 2015). Urbanization and industrialization coupled with anthropogenic influences have resulted in forest degradation at an alarming rate across the globe (Sarmah et al., 2011; Nagendra and Utkarsch, 2003). In Pakistan, montane temperate forests have coniferous vegetation with sparsely distributed broad-leaved species (Hussain and Illahi, 1991). Altay et al. (2012) recorded the plant communities in 
urban habitats of Istanbul, Turkey. They documented 13 plant communities and studied the impact of physico-chemical characteristics of soil on these vegetation types. Digiovinazzo et al. (2010) reported that phytosociological thresholds provide guidelines for conservation strategies. Wahab et al. (2008) assessed vegetation structure at 5 monitoring sites of Dangam District, Afghanistan. Vegetation structure of Amazonian Ecuador was studied by Maestre et al. (2006). In Mwanihana forests of Tanzania, Lovett et al. (2006) used phytosociological techniques to conclude that higher species diversity is found at high altitudes. Gould et al. (2006) assessed the structure and composition of vegetation along an elevation gradient in Puerto Rico. Angassa (2005) documented shrubby and grass vegetation in Borna Rangeland ecosystem. Dondeyne et al. (2004) studied the vegetation and soil attributes in woodlands of Southeastern Tanzania. They reported profound impact of edaphic variables on vegetation structure. Vegetation structure of Maasai Mara National Reserve was studied by Salvatori et al. (2003). They also explained the impact of fire and grazing on vegetation structure. Luis et al. (2002) recognized two major vegetation types in Cufada Lagoon in GuineaBissau. Grillini et al. (2001) carried out phytosociological studies in Chambura Gorge, Queen Elizabith National Park. Some phytosociological works from Pakistan are presented here. Urooj et al. (2016) conducted vegetation analysis on wetland area around Mangla Dam, Azad Kashmir. Noor and Khatoon (2013) studied vegetation patterns and soil attributes in Astor Valley, Gilgit Baltistan. Shaheen and Qureshi (2011) assessed vegetation of Lesser Himalayan Subtropical forests in Bagh, Azad Kashmir. They also recorded anthropogenic impacts on vegetation structure. Phytosociology and soil characteristics of Quercus forests of Chitral was studied by Khan et al. (2010). Siddiqui et al. (2010) documented the phytosociology of moist temperate Himalayan forests in Pakistan. They classified the vegetation into 3 major groups. Ahmad et al. (2010) presented a review on status of vegetation analysis in Pakistan. Hussain et al. (2009) recorded plant communities from Nurpur reserved forest, District Chakwal. Wazir et al. (2008) identified 5 vegetation types in Chapursan Valley, Gilgit. Ahmad et al. (2008) studied the spatio-temporal effects on plant associations in Soone Valley. From Khyber Pakhtunkhwa province studies of community ecology are summed up here. Ilyas et al. (2015) studied the vegetation structure of Kabal valley, Swat. Wahab et al. (2010) studied vegetation structure in 25 forest types in District Dir. Shah and Hussain (2009) documented the vegetation structure in Hayatabad, Peshawar, establishing 5 plant communities. Hussain et al. (2005) described 7 plant communities from Ghalegy hills, Swat. Hussain and Badshah (1998) documented the vegetation of Pirghar hills, South Waziristan. Review of literature clearly indicates that no comprehensive study is available on vegetation structure of Chail, Shinku, Bishigram and Shinku, hence present study will fill the research gap in these areas which have rich floral diversity. Present study was carried out to map vegetation and to assess the threats to montane temperate forests in Hindukush range, Swat, Pakistan.

\section{Materials and methods}

\section{Study area}

The research work was carried out in Hindukush range, District Swat. Swat is located from $34^{\circ} 34^{\prime}$ to $35^{\circ} 55^{\prime}$ North latitudes and $72^{\circ} 08^{\prime}$ to $72^{\circ} 50^{\prime}$ East longitudes. The study area spreads over an area of 24148 acres, including villages of Chail, Shinku, 
Dabargai and Bishigram (Fig. 1). Most part of the area falls under moist temperate zone with typical flora and fauna.
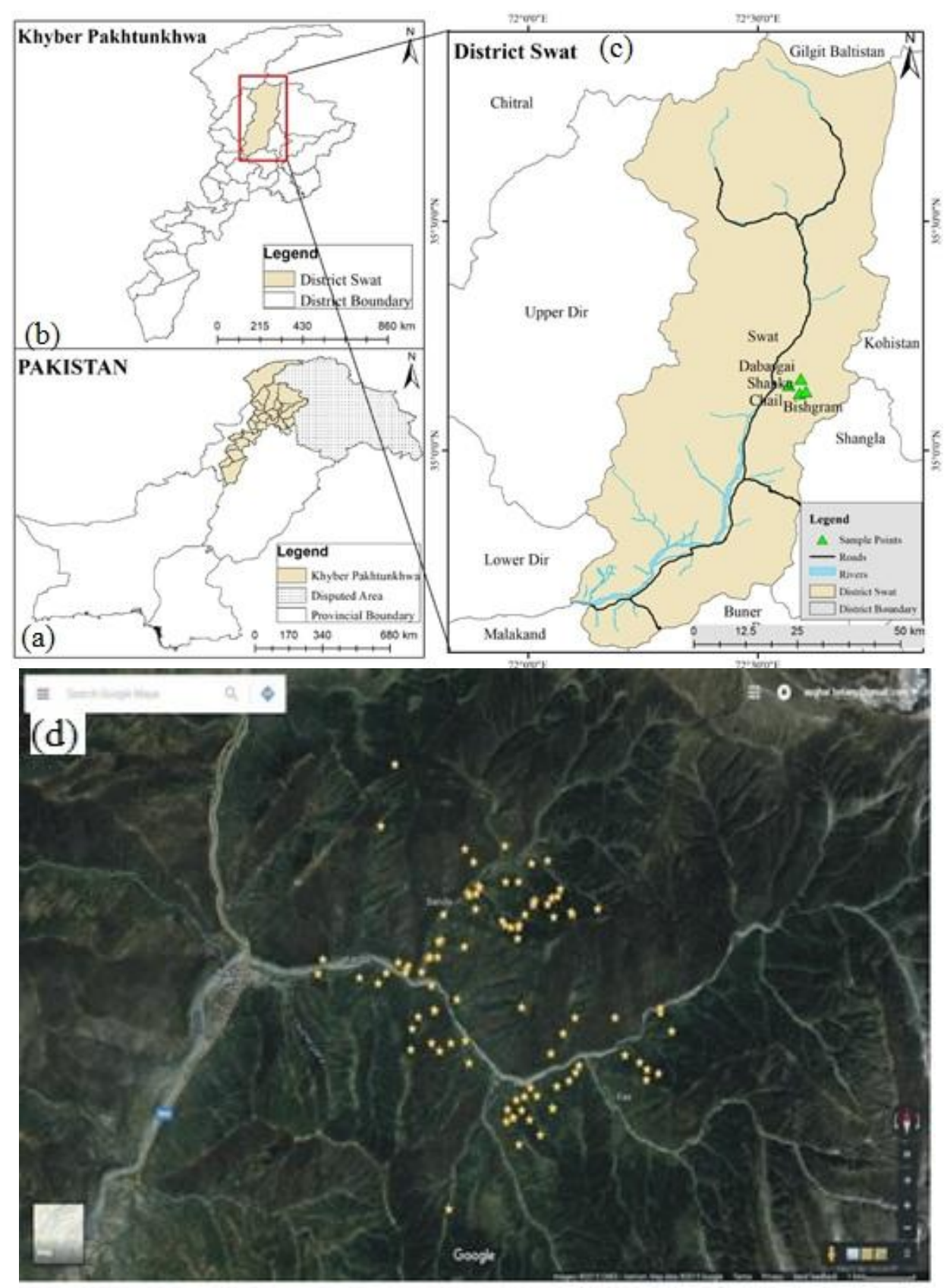

Figure 1. (a) Map of Pakistan. (b) Map of Khyber Pakhtunkhwa Province. (c) Enlarged map of District Swat and monitoring sites. (d) Map of the study area showing all the sampling sites

\section{Phytosociology}

Phytosociological studies were conducted in 5 monitoring sites. These sites were chosen based on species composition, habitats, and physiognomic contrast. Vegetation was analyzed by using $5(10 \times 10 \mathrm{~m})$ quadrats for trees, $10(5 \times 5 \mathrm{~m})$ quadrats for shrubs and $15(1 \times 1 \mathrm{~m})$ quadrats for herbs at each monitoring site. Density, cover and 
frequency of each species were measured and values were changed to relative values to calculate IV (Importance value) and FIV (Family importance value). The plant communities were established based on highest importance values following Ahamd and Shaukat (2012), Hussain (1989).

\section{Maturity index}

Community maturity index was obtained by Pichi-Sermolli (1948) method.

\section{Similarity index}

Similarity index was calculated by using Sorensen's index (Sorensen, 1948) as modified by Motyka et al. (1950).

\section{Soil analysis}

Standard methods were applied to estimate Soil texture (Brady, 1990; Bouyoucos, 1936), pH (Jackson, 1962), Organic matter, $\mathrm{CaCO}_{3}$ (Rayan et al., 1997), Electrical conductivity (Rhoades, 1996), Sodium absorption ratio and Sulphates (Richard, 1954). Nitrogen was determined by Kieldahl method of Bremner and Mulvaney (1982). Phosphorous, Potassium, Calcium and Magnesium were enumerated by Inductively Coupled Plasma Optical Emission Spectroscopy (ICP-OES).

\section{Results}

Present study was conducted in Hindukush range of District Swat, Pakistan during 2013-2017. Five monitoring sites viz. Lower Chail, Upper Chail, Shinku, Dabargai and Bishigram were established. The study area displays a unique floristic and vegetation structure. A total of 202 species were recorded in sampling units, of which trees were represented by 33 species, shrubs by 36 species and herbs by 133 species. Based on importance value (IV), three communities respectively of trees, shrubs and herb layer were established at each monitoring site. Tree communities comprised of 16 families, with Pinnaceae as the leading family with an FIV of 190.85 followed by Fagaceae (64.97), Juglandaceae (45.51), Ebenaceae (38.54), Moraceae (31.64). Rosaceae (30.41), Simoroubaceae (22.36) and Salicaceae with 19.86. Oleaceae, Taxaceae, Punicaceae, Meliaceae and Ulmaceae had importance values ranging from 16.8 to 5.13. Shrubby vegetation was represented by 21 families, dominated by Rosaceae, with highest FIV value of 101.44. It was followed by Papilionaceae (59.27), Hammelidaceae (41.62), Berberidaceae (33.47), Thymelaceae (33.44), Oleaceae (31.91), Buxaceae (30.21), Myrsinaceae (27.14), Lamiaceae (26.16) and Caprifoliaceae (23.43). Similarly, Buddlejaceae, Euphorbiaceae, Elaeagnaceae, Grossulariaceae, Rhamnaceae, Acanthaceae, Urticaceae, Rutaceae and Plumbaginaceae were the associated families having FVI ranging from 16.98 to 4.96 . Herbaceous layers were represented by 47 families with Poaceae as the leading family with FIV value of 182.15. Asteraceae (43.06), Lamiaceae (27.55), Papilionaceae (24.06), Polygonaceae (23.15), Saxifragaceae (17.4), Brassicaceae (14.24), Euphorbiaceae (14.09), Rosaceae (13.82) and Apiaceae (12.35) were the sub dominant families. Other important families of herbaceous layer included Plantaginaceae, Ranunculaceae, Caryophyllaceae, Sapotaceae, Solanaceae, Amaryllidaceae, Scrophulariaceae, Urticaceae and Oxalidaceae (Tables 1 and 2). 
Table 1. Family importance values

\begin{tabular}{|c|c|c|c|c|c|}
\hline \multirow{2}{*}{ S.No } & TREES & \multirow{2}{*}{ FIV } & \multirow{2}{*}{ S.No } & \multirow{2}{*}{ Family } & \multirow{2}{*}{ FIV } \\
\hline & Family & & & & \\
\hline 1 & Pinaceae & 190.85 & 5 & Polygonaceae & 23.15 \\
\hline 2 & Fagaceae & 64.97 & 6 & Saxifragaceae & 17.4 \\
\hline 3 & Juglandaceae & 45.51 & 7 & Brassicaceae & 14.24 \\
\hline 4 & Ebenaceae & 38.54 & 8 & Euphorbiaceae & 14.09 \\
\hline 5 & Moraceae & 31.64 & 9 & Rosaceae & 13.82 \\
\hline 6 & Rosaceae & 30.41 & 10 & Apiaceae & 12.35 \\
\hline 7 & Simaroubaceae & 22.36 & 11 & Plantaginaceae & 9.95 \\
\hline 8 & Salicaceae & 19.86 & 12 & Ranunculaceae & 9.88 \\
\hline 9 & Oleaceae & 16.8 & 13 & Caryophyllaceae & 9.43 \\
\hline 10 & Taxaceae & 15.73 & 14 & Sapotaceae & 8.54 \\
\hline 11 & Punicaceae & 5.91 & 15 & Solanaceae & 8.01 \\
\hline 12 & Meliaceae & 5.82 & 16 & Amaryllidaceae & 7.62 \\
\hline 13 & Ulmaceae & 5.13 & 17 & Scrophulariaceae & 6.8 \\
\hline 14 & Plantanaceae & 3.6 & 18 & Urticaceae & 5.99 \\
\hline 15 & Aceraceae & 3.07 & 19 & Oxalidaceae & 5.97 \\
\hline 16 & Anacardiaceae & 2.39 & 20 & Cannabaceae & 5.54 \\
\hline S.No & SHRUBS & FIV & 21 & Gentianaceae & 5.5 \\
\hline 1 & Rosaceae & 101.44 & 22 & Colchicaceae & 4.38 \\
\hline 2 & Papilionaceae & 59.27 & 23 & Chenopodiaceae & 3.67 \\
\hline 3 & Hammelidaceae & 41.62 & 24 & Rubiaceae & 3.39 \\
\hline 4 & Berberidaceae & 33.47 & 25 & Amaranthaceae & 3.14 \\
\hline 5 & Thymelaceae & 33.44 & 26 & Covolvulaceae & 3.13 \\
\hline 6 & Oleaceae & 31.91 & 27 & Malvaceae & 2.82 \\
\hline 7 & Buxaceae & 30.21 & 28 & Cucutaceae & 2.68 \\
\hline 8 & Myrsinaceae & 27.14 & 29 & Primulaceae & 2.35 \\
\hline 9 & Lamiaceae & 26.16 & 30 & Papaveraceae & 2.16 \\
\hline 10 & Caprifoliaceae & 23.43 & 31 & Iridaceae & 2.08 \\
\hline 11 & Euphorbiaceae & 16.98 & 32 & Campanulaceae & 1.79 \\
\hline 12 & Buddlejaceae & 15.65 & 33 & Liliaceae & 1.7 \\
\hline 13 & Elaeagnaceae & 12.16 & 34 & Podophyllaceae & 1.66 \\
\hline 14 & Grossulariaceae & 8.59 & 35 & Boraginaceae & 1.62 \\
\hline 15 & Rhamnaceae & 7.6 & 36 & Hypericaceae & 1.53 \\
\hline 16 & Acanthaceae & 7.09 & 37 & Fumariaceae & 1.02 \\
\hline 17 & Urticacaeae & 6.21 & 38 & Violaceae & 0.92 \\
\hline 18 & Rutaceae & 5.75 & 39 & Crassulaceae & 0.78 \\
\hline 19 & Plumbaginaceae & 4.96 & 40 & Valerianaceae & 0.78 \\
\hline 20 & Celastraceae & 4.63 & 41 & Verbenaceae & 0.78 \\
\hline 21 & Rubiaceae & 1.89 & 42 & Portulacaceae & 0.7 \\
\hline S.No & HERBS & FIV & 43 & Zygophyllaceae & 0.51 \\
\hline 1 & Poaceae & 182.15 & 44 & Orchidaceae & 0.5 \\
\hline 2 & Asteraceae & 43.06 & 45 & Dioscoraceae & 0.46 \\
\hline 3 & Lamiaceae & 27.55 & 46 & Gereniaceae & 0.42 \\
\hline 4 & Papilionaceae & 24.06 & 47 & Paeoniaceae & 0.42 \\
\hline
\end{tabular}


Table 2. IV data for plant species

\begin{tabular}{|c|c|c|c|c|c|c|c|c|c|c|c|c|c|}
\hline & & $\mathrm{LC}$ & UC & $\mathrm{S}$ & $\mathrm{D}$ & B & & & $\mathrm{LC}$ & $\mathrm{UC}$ & $\mathrm{S}$ & $\mathrm{D}$ & $\mathrm{B}$ \\
\hline & Trees & PDQ & PQJ & QAP & CAP & PPA & & Trees & PDQ & PQJ & QAP & CAP & PPA \\
\hline 1 & Abies pindrow Royle. & - & - & 18.6 & 16.01 & 12.36 & 23 & Prunus persica (L.) Batsch. & - & 3.93 & - & - & - \\
\hline 2 & Acer cappadocicum Gled. & - & - & - & - & 3.07 & 24 & Punica granatum $\mathrm{L}$. & - & 5.91 & - & - & - \\
\hline 3 & Ailanthus altissima (Mill.) Swingle & 9.21 & 6.42 & 1.89 & 2.37 & 2.47 & 25 & Pyrus pashia Ham. ex D. Don & - & - & 4.16 & - & - \\
\hline 4 & Cedrela serrata Royle & - & - & - & 2.91 & - & 26 & Pyrus pyrifolia (Burm.) Nak. & - & - & 4.01 & - & 2.56 \\
\hline 5 & $\begin{array}{l}\text { Cedrus deodara (Roxb. ex Lamb.) } \\
\text { G. Don }\end{array}$ & - & - & 4.02 & 22.07 & 2.92 & 27 & Quercus baloot Griffth & - & 15.52 & - & 3.88 & - \\
\hline 6 & Celtis caucasica Willd. & - & - & - & 2.62 & 2.51 & 28 & Quercus dilatata Royle & 15.62 & - & - & 3.02 & 2.79 \\
\hline 7 & Crataegus songarica G. Koch. & 4.49 & 3.21 & 2.04 & 2.47 & - & 29 & Quercus incana Roxb. & - & - & 18.91 & - & 2.47 \\
\hline 8 & Diospyros kaki L. & - & - & 3.64 & - & - & 30 & Quercus semecarpifolia Sm. & - & - & - & - & 2.76 \\
\hline 9 & Diospyros lotus L. & 23.38 & 2.87 & 1.86 & 2.71 & 4.12 & 31 & Salix babylonica L. & - & - & - & 2.47 & - \\
\hline 10 & Ficus carica L. ssp carica & 8.94 & 4.23 & 1.76 & 4.41 & 3.6 & 32 & Salix denticulata Anderson & - & 6.66 & 4.19 & - & 2.61 \\
\hline 11 & Juglans regia $\mathrm{L}$. & 12.93 & 13.36 & 11.59 & 3.27 & 4.36 & 33 & Taxus fauana (Zucc.) Pilger & - & - & 8.88 & 4.06 & 2.79 \\
\hline 12 & Malus pumila Mill. & - & 3.54 & - & - & - & & Shrubs & ICJ & RIR & SDB & PVB & PCI \\
\hline 13 & Melia azedarach $\mathrm{L}$. & - & - & - & 2.91 & - & 1 & $\begin{array}{l}\text { Andrachne cordifolia (Wall.ex } \\
\text { Dcne.) Muell. Arg. }\end{array}$ & - & - & - & 2.1 & 3.25 \\
\hline 14 & Morus alba $\mathrm{L}$. & - & - & 1.81 & 3.02 & - & 2 & Berberis lycium Royle & - & 3.67 & 13.6 & 12.26 & 3.94 \\
\hline 15 & Morus nigra $\mathrm{L}$. & - & 3.87 & - & - & - & 3 & Buddleja crispa Bth. & 9.6 & - & 6.05 & - & - \\
\hline 16 & Olea ferruginea Royle & - & 8.42 & - & 5.53 & 2.85 & 4 & $\begin{array}{l}\text { Cotoneaster nummularia Fisch. \& } \\
\text { Mey. }\end{array}$ & 13.25 & - & 7.3 & 2.47 & 14.82 \\
\hline 17 & Picea smithiana (Wall.) Boiss. & - & - & - & 2.8 & 22.07 & 5 & Daphne mucronata Royle & 6.84 & 5.49 & 17.29 & 1.96 & 1.86 \\
\hline 18 & Pinus roxburghii Sargent & - & - & - & - & 3.07 & 6 & $\begin{array}{l}\text { Debregeasia salicifolia (D. Don) } \\
\text { Rendle }\end{array}$ & - & 6.21 & - & - & - \\
\hline 19 & Pinus wallichiana A.B. Jackson & 25.39 & 18.06 & 12.56 & 13.38 & 14.54 & 7 & Elaeagnus umbellate Thunb. & 6.31 & - & 3.93 & - & 1.92 \\
\hline 20 & $\begin{array}{l}\text { Pistacia chinensis Bunge } s s p . \\
\text { Integerrima }\end{array}$ & - & - & - & - & 2.39 & 8 & $\begin{array}{l}\text { Himalrandia tetrasperma (Roxb.) } \\
\text { Yamazaki }\end{array}$ & - & - & - & - & 1.89 \\
\hline 21 & Platanus orientalis $\mathrm{L}$. & - & - & - & - & 3.6 & 9 & $\begin{array}{l}\text { Indigofera heterantha Wall. Ex } \\
\text { Brandis var. gerardiana }\end{array}$ & 13.36 & 7.31 & - & 7.08 & - \\
\hline 22 & Populus alba L. & - & 3.93 & - & - & - & 10 & $\begin{array}{l}\text { Indigofera heterantha Wall. ex } \\
\text { Brandis var. heterantha }\end{array}$ & - & - & 10.24 & 2.1 & 11.95 \\
\hline
\end{tabular}




\begin{tabular}{|c|c|c|c|c|c|c|c|c|c|c|c|c|c|}
\hline & & $\mathrm{LC}$ & $\mathrm{UC}$ & $\mathrm{S}$ & $\mathrm{D}$ & B & & & LC & $\mathrm{UC}$ & $S$ & $\mathrm{D}$ & B \\
\hline & Shrubs & ICJ & RIR & SDB & PVB & PCI & & Shrubs & ICJ & RIR & SDB & PVB & PCI \\
\hline 11 & Isodon rugosus (Wall. ex Bth.) Codd & - & 13.67 & - & 3.86 & 3.44 & 33 & Spiraea canescens D. Don & 10.01 & - & - & - & 4.47 \\
\hline 12 & Jasminum humile $\mathrm{L}$. & 10.23 & - & 6.68 & 3.46 & 4.6 & 34 & Strobilanthes urticifolia Wall. ex Kuntze & - & - & - & 2.41 & - \\
\hline 13 & Jasminum officinale $L$. & - & 6.94 & - & - & - & 35 & Viburnum cotinifolium D. Don & 4.81 & - & - & - & - \\
\hline 14 & Justicia adhatoda $\mathrm{L}$. & - & 4.68 & - & - & - & 36 & Viburnum grandiflorum Wall. ex DC. & - & - & - & 13.08 & - \\
\hline 15 & Lespedeza juncea (L. f.) Pers. var. juncea & - & 3.89 & - & - & 3.34 & & Herbs & $\mathrm{ACH}$ & AHP & $\mathrm{CAB}$ & CTC & CMR \\
\hline 16 & Limonium cabulicum (Boiss.) O. Kuntze & - & - & - & - & 4.96 & 1 & Achillea millefolium $\mathrm{L}$. & - & 1.09 & - & - & - \\
\hline 17 & Lonicera asperifolia (Decne.) & - & 3.62 & - & - & - & 2 & $\begin{array}{l}\text { Achyranthes aspera L. var. pubescens } \\
\text { (Moq.) C.C. Townsend }\end{array}$ & - & - & - & 0.42 & - \\
\hline 18 & $\begin{array}{l}\text { Lonicera obovata Royle ex Hk. f. \& } \\
\text { Thoms. }\end{array}$ & - & - & - & 1.92 & - & 3 & Agrimonia eupatoria L. & - & - & - & - & 0.42 \\
\hline 19 & $\begin{array}{l}\text { Maytenus royleanus (Wall. ex Lawson) } \\
\text { Cuf. }\end{array}$ & - & - & - & 4.63 & - & 4 & Agrostis viridis Gouan & - & - & - & 1.99 & 0.5 \\
\hline 20 & Myrsine africana $\mathrm{L}$. & 7.34 & 8.9 & 6.62 & 4.28 & - & 5 & Ajuga bracteosa Wall. ex Bth. & - & - & - & 1.08 & - \\
\hline 21 & $\begin{array}{l}\text { Parrotiopsis jacquemontiana (Dcne.) } \\
\text { Rehder }\end{array}$ & - & - & - & 25.51 & 16.11 & 6 & Ajuga parviflora Bth. & - & - & - & - & 0.74 \\
\hline 22 & Phlomis spectabilis Falc. ex Bth & - & - & - & 1.93 & 3.26 & 7 & Amaranthus viridis $\mathrm{L}$. & - & 1.69 & - & 1.03 & - \\
\hline 23 & Ribes himalense Dcne. & - & - & - & 4.05 & - & 8 & Ammi visnaga (L.) Lam. & - & - & - & 0.51 & - \\
\hline 24 & Ribes orientale Desf. & - & - & - & - & 4.54 & 9 & $\begin{array}{l}\text { Anagallis arvensis L. var. coerulea (L.) } \\
\text { Gouan. }\end{array}$ & - & - & 1.31 & 0.42 & 0.63 \\
\hline 25 & Ricinus communis L. & - & 11.63 & - & - & - & 10 & Angelica glauca Edgew. & - & - & - & - & 1.19 \\
\hline 26 & Rosa webbiana Wall. ex Royle & - & - & 3.51 & 1.96 & 1.55 & 11 & Arthraxon prionodes (Steud.) Dandy & 3.61 & - & - & - & - \\
\hline 27 & Rubus ellipticus Smith & 6.43 & - & - & - & - & 12 & Apluda mutica $\mathrm{L}$. & 14.57 & 9.81 & 12.47 & 1.85 & 0.54 \\
\hline 28 & Rubus fruiticosus $\mathrm{L}$. & 7.23 & - & 5.71 & 1.97 & 4.84 & 13 & Arenaria serpyllifolia $\mathrm{L}$. & - & - & - & - & 1.07 \\
\hline 29 & Rubus ulmifolius Schott & - & 15.92 & - & - & - & 14 & Aristida cyanantha Nees ex Steud. & - & - & - & - & 3.12 \\
\hline 30 & $\begin{array}{l}\text { Sageretia thea (Osbeck) M.C. Johnston } \\
\text { var. thea }\end{array}$ & 4.52 & - & - & - & 3.08 & 15 & Artemisia absinthium $\mathrm{L}$. & - & - & - & 2.36 & - \\
\hline 31 & Sarcococca saligna (D. Don) Muell.Arg. & - & 8 & 19.01 & - & 3.2 & 16 & Artemisia scoparia Waldst. \& Kit. & - & 5.19 & - & - & - \\
\hline 32 & $\begin{array}{l}\text { Skimmia laureola (DC.) Sieb. \& Zucc. ex } \\
\text { Walp }\end{array}$ & - & - & - & 2.88 & 2.87 & 17 & Artemisia vulgaris $\mathrm{L}$. & 4.93 & - & 6.46 & - & - \\
\hline
\end{tabular}




\begin{tabular}{|c|c|c|c|c|c|c|c|c|c|c|c|c|c|}
\hline & & $\mathrm{LC}$ & $\mathrm{UC}$ & $\mathrm{S}$ & $\mathrm{D}$ & B & & & $\mathrm{LC}$ & $\mathrm{UC}$ & $\mathrm{S}$ & $\mathrm{D}$ & B \\
\hline & Herbs & $\mathrm{ACH}$ & AHP & $\mathrm{CAB}$ & CTC & CMR & & Herbs & $\mathrm{ACH}$ & AHP & $\mathrm{CAB}$ & CTC & CMR \\
\hline 18 & Avena fatua $L$. & - & - & 3.53 & 3.58 & - & 40 & Convolvulus arvensis $L$. & - & 1.42 & - & 0.93 & 0.78 \\
\hline 19 & $\begin{array}{l}\text { Bergenia ciliata (Haw.) Sternb. var. } \\
\text { ciliata Yeo }\end{array}$ & 5.07 & - & 6.88 & 3.09 & 1.29 & 41 & Conyza Canadensis (L.) Crongquist & - & 1.02 & - & 0.93 & - \\
\hline 20 & $\begin{array}{l}\text { Bistorta amplexicaulis (D. Don) } \\
\text { Green }\end{array}$ & 2.54 & - & - & 0.93 & - & 42 & Cuscuta europaea $L$. & 1.39 & - & 0.42 & - & - \\
\hline 21 & $\begin{array}{l}\text { Brachiaria reptans (L.) Gardner \& } \\
\text { Hubbard }\end{array}$ & - & - & - & - & 0.42 & 43 & Cuscuta reflexa Roxb. & - & 0.87 & - & - & - \\
\hline 22 & Brassica rapa L. ssp. Campestris & - & 2.09 & - & - & - & 44 & Cynodon dactylon (L.) Pers. & 13.35 & - & 13.97 & 8.64 & 3.4 \\
\hline 23 & Buplerum falcatum $L$. & 2.2 & - & 2.03 & - & - & 45 & Dactylis glomerata $L$. & - & - & - & 0.42 & 0.42 \\
\hline 24 & $\begin{array}{l}\text { Bupleurum longicaule Wall. ex DC. } \\
\text { var. ramosum Nasir }\end{array}$ & - & - & - & 0.98 & - & 46 & Datura innoxia Mill. & - & 1.63 & - & - & - \\
\hline 25 & Calanthe tricarinata Lindl. & - & - & - & - & 0.5 & 47 & Dicanthium annulatum (Forssk.) Stapf & - & - & - & 0.46 & - \\
\hline 26 & Caltha alba Camb. var. alba & - & - & 0.66 & 2.11 & - & 48 & Dioscorea deltoidea Wall. ex Kunth & - & - & - & 0.46 & - \\
\hline 27 & Campanula pallida Wall. var. pallida & - & 1.79 & - & - & - & 49 & Eragrostis minor Host & - & - & - & - & 1.31 \\
\hline 28 & Cannabis sativa $L$. & - & 1.78 & - & 2.39 & 1.37 & 50 & Erodium ciconium (L.) L' Herit ex Aiton & - & - & - & - & 0.42 \\
\hline 29 & Capsella bursa-pastoris (L.) Medik. & - & 1.67 & - & 1.67 & 1.44 & 51 & Eryngium coeruleum M-Bieb. & - & 1.74 & - & - & 1.33 \\
\hline 30 & $\begin{array}{l}\text { Cenchrus pennisetiformis Hochst. \& } \\
\text { Steud. Ex Steud. }\end{array}$ & - & - & - & - & 18.92 & 52 & Euphorbia helioscopia L. & - & - & 1.59 & - & 0.78 \\
\hline 31 & Centaurea iberica Trev. ex Spreng. & - & - & - & 0.42 & - & 53 & Euphorbia peplus $L$. & - & 1.81 & - & 2.18 & 0.46 \\
\hline 32 & Ceratocephala falcata (L.) Pers. & - & - & - & - & 0.42 & 54 & Euphorbia prostrata Ait. & 2.75 & - & 1.44 & 0.79 & - \\
\hline 33 & Chenopodium album $L$. & - & 2.83 & - & 0.84 & - & 55 & Foeniculum vulgare Mill. & - & 0.47 & - & - & - \\
\hline 34 & Chrysopogon fulvus (Spreng.) Chiov. & - & - & - & 10.89 & - & 56 & Fragaria vesca $L$. & 5.82 & - & 6.74 & 0.42 & - \\
\hline 35 & Chrysopogon serrulatus Trin. & - & - & - & - & 0.42 & 57 & Fumaria indica (Hausskn.) Pugsley & - & 0.47 & - & 0.55 & - \\
\hline 36 & Cichorium intybus $L$. & 1.32 & - & 1.12 & 0.88 & - & 58 & Gagea elegans Wall. ex Royle & - & - & - & 0.42 & - \\
\hline 37 & Clematis grata Wall. & 2.53 & - & 2.21 & - & - & 59 & Galium aparine $L$. & - & 2.61 & - & - & 0.78 \\
\hline 38 & $\begin{array}{l}\text { Clinopodium umbrosum (M. Bieb.) } \\
\text { C. Koch. }\end{array}$ & 3.68 & - & - & 0.55 & - & 60 & $\begin{array}{l}\text { Heliotropium strigosum Willd. ssp. } \\
\text { brevifolium (Wall.) Kazmi }\end{array}$ & - & - & - & - & 0.74 \\
\hline 39 & Colchicum luteum Baker & - & 1.94 & - & 1.37 & 1.07 & 61 & Heteropogon contortis $L$. & 6.64 & 8.34 & - & - & - \\
\hline
\end{tabular}




\begin{tabular}{|c|c|c|c|c|c|c|c|c|c|c|c|c|c|}
\hline & & $\mathrm{LC}$ & $\mathrm{UC}$ & $S$ & $\mathrm{D}$ & B & & & LC & $\mathrm{UC}$ & $S$ & $\mathrm{D}$ & B \\
\hline & Herbs & $\mathrm{ACH}$ & AHP & $\mathrm{CAB}$ & CTC & CMR & & Herbs & $\mathrm{ACH}$ & AHP & $\mathrm{CAB}$ & CTC & CMR \\
\hline 62 & Hypericum oblongifolium Choisy & - & - & 0.23 & - & - & 84 & Oxalis corniculata $\mathrm{L}$. & - & - & 2.41 & - & 0.82 \\
\hline 63 & Hypericum perforatum $\mathrm{L}$. & - & - & 0.42 & 0.88 & - & 85 & Paeonia emodi Wall. ex Royle & - & - & & 0.42 & - \\
\hline 64 & Iris germanica $\mathrm{L}$. & - & - & - & - & 1.11 & 86 & Papaver dubium $\mathrm{L}$. & - & - & - & - & 1.19 \\
\hline 65 & Iris hookeriana Foster & - & - & - & 0.97 & - & 87 & Papaver somniferum L. & - & 0.97 & - & - & - \\
\hline 66 & Lactuca orientalis Boiss. & - & - & - & 0.46 & - & 88 & Pennisetum orientale L.C. Rich. & 1.96 & 2.62 & - & - & - \\
\hline 67 & Lepidium apetalum Willd. & - & - & - & - & 1.03 & 89 & Phalaris minor Retz. & - & 6.54 & - & 2.13 & - \\
\hline 68 & Lepidium sativum $\mathrm{L}$. & - & - & - & 0.42 & - & 90 & Plantago lanceolata $\mathrm{L}$. & - & - & 0.42 & 1.3 & 1.81 \\
\hline 69 & Lolium temulentum $\mathrm{L}$. & - & - & - & 1.3 & - & 91 & Plantago himalaica Pilger & 2.37 & - & - & 0.46 & 0.7 \\
\hline 70 & Notholirion thomsonianum (Royle) Stapf & - & - & - & 0.46 & - & 92 & Plantago major $\mathrm{L}$. & - & 2.89 & - & - & - \\
\hline 71 & Malva neglecta Wall. & - & - & - & - & 0.74 & 93 & Pоа аппиа $\mathrm{L}$. & - & 7.92 & 4.22 & - & 3.8 \\
\hline 72 & Malvastrum coromendelianum $\mathrm{L}$. & - & - & 0.42 & 0.42 & 1.24 & 94 & Podophyllum emodi Wall. ex Royle & - & - & - & - & 1.66 \\
\hline 73 & Medicago lupulina $\mathrm{L}$. & - & - & - & - & 11.87 & 95 & Portulaca oleracea $\mathrm{L}$. & - & - & - & - & 0.7 \\
\hline 74 & Medicago minima (L.) Grufb. & - & 2.51 & - & 0.69 & - & 96 & Pseudognaphalium affine (D. Don.) Anderb. & - & - & - & - & 0.46 \\
\hline 75 & Medicago polymorpha $\mathrm{L}$. & 2.92 & 1.79 & 0.66 & - & 0.99 & 97 & Ranunculus laetus Wall. ex Hk. f. \& Thoms. & - & 1.44 & - & - & - \\
\hline 76 & Mentha longifolia $(\mathrm{L}.) \mathrm{L}$. & - & 2.34 & - & - & - & 98 & Ranunculus sceleratus $\mathrm{L}$. & - & - & - & - & 0.78 \\
\hline 77 & $\begin{array}{l}\text { Micromeria biflora (Buch-Ham.ex D. } \\
\text { Don) Bth. }\end{array}$ & - & - & - & 0.46 & - & 99 & Rumex dentatus $\mathrm{L}$. & - & - & 0.84 & 1.11 & - \\
\hline 78 & $\begin{array}{l}\text { Myosotis alpestris F. W. Schmidt var. } \\
\text { albicans (H. Riedl) Y. Nasir }\end{array}$ & - & - & - & - & 0.46 & 100 & Rumex hastatus D. Don & 2.45 & - & 4.63 & 1.68 & 8.97 \\
\hline 79 & Narcissus tazetta $\mathrm{L}$. & - & 2.56 & 3.16 & 1.12 & 0.78 & 101 & Salvia lanata Roxb. & - & 1.74 & - & 0.51 & - \\
\hline 80 & Oenanthe javanica (Blume) DC. & - & - & - & 0.42 & - & 102 & Salvia moorcraftiana Wall. Ex Bth. & 3.32 & - & 2.43 & 3.04 & - \\
\hline 81 & Onosma hispida Wall. ex G. Don & - & - & - & 0.42 & - & 103 & Sanguisorba minor Scop. & - & - & - & 0.42 & - \\
\hline 82 & Origanum vulgare $\mathrm{L}$. & 4.3 & - & - & - & - & 104 & Saussurea albescens (DC.) Sch. Bip. & 3.92 & - & 4.11 & 0.51 & - \\
\hline 83 & Oxalis acetosella $L$. & - & 1.09 & - & 0.74 & 0.91 & 105 & Saxifraga moorcroftiana (Ser.) Sternb. & - & - & - & - & 1.07 \\
\hline
\end{tabular}




\begin{tabular}{|c|c|c|c|c|c|c|c|c|c|c|c|c|c|}
\hline & & $\mathrm{LC}$ & $\mathrm{UC}$ & $\mathrm{S}$ & $\mathrm{D}$ & B & & & $\mathrm{LC}$ & UC & $\mathrm{S}$ & $\mathrm{D}$ & B \\
\hline & Herbs & $\mathrm{ACH}$ & AHP & $\mathrm{CAB}$ & CTC & CMR & & Herbs & $\mathrm{ACH}$ & AHP & $\mathrm{CAB}$ & CTC & CMR \\
\hline 106 & Scandix pecten-veneris $\mathrm{L}$. & - & - & 1.48 & - & - & 120 & $\begin{array}{l}\text { Teucrium stocksianum Boiss. var. } \\
\text { incanum (Aitch. \& Hemsley) Hedge \& } \\
\text { Lamon }\end{array}$ & - & 2.81 & - & - & - \\
\hline 107 & Sedum hispanicum $\mathrm{L}$. & - & - & - & - & 0.78 & 121 & $\begin{array}{l}\text { Themeda anathera (Nees ex Steud.) } \\
\text { Hack. }\end{array}$ & - & - & - & 9.33 & - \\
\hline 108 & Silene conoidea $\mathrm{L}$. & - & - & 2.05 & - & - & 122 & Thymus linearis Bth. ssp. linearis Jalas & - & - & - & 0.55 & - \\
\hline 109 & Silene viscosa (L.) Pers. & - & - & - & 0.93 & 0.46 & 123 & Tragopogon gracilis D.Don & - & - & - & - & 0.42 \\
\hline 110 & Silene vulgaris (Moench) Garcke & - & 1.49 & - & - & - & 124 & Tribulus terrestris L. & - & - & - & 0.51 & - \\
\hline 111 & Sisymbrium irio L. & - & - & - & 2.46 & 2.83 & 125 & Trigonella emodi Bth. & - & - & - & - & 0.46 \\
\hline 112 & Solanum nigrum L. var. nigrum & - & 1.42 & 0.7 & 0.88 & 1.98 & 126 & Tulipa stellata $\mathrm{Hk}$. f. & - & - & - & - & 0.82 \\
\hline 113 & Solanum pseudocapcicum L. & - & - & 0.66 & - & - & 127 & Urtica dioca $\mathrm{L}$. & - & 3.81 & - & - & 2.18 \\
\hline 114 & Solidago virga-aurea $\mathrm{L}$. & 2.13 & - & 2.99 & 0.55 & - & 128 & Valeriana jatamansi Jones & - & - & - & - & 0.78 \\
\hline 115 & Sonchus asper (L.) Hill & 2.62 & - & 3.03 & 0.93 & 0.42 & 129 & Verbascum thapsus L. & - & 3.71 & - & 3.09 & - \\
\hline 116 & Stellaria media (L.) Vill. & - & - & 1.03 & 1.9 & 0.5 & 130 & Verbena officinalis $\mathrm{L}$. & - & - & - & - & 0.78 \\
\hline 117 & Swertia ciliata (G. Don) B. L. Burtt & 3.54 & 0.99 & - & - & - & 131 & Vicia monantha Retz. & - & - & 1.33 & 0.42 & 0.42 \\
\hline 118 & Swertia cordata (G. Don) Clarke & - & - & - & 0.97 & - & 132 & Viola canescens Wall. ex Roxb. & - & 0.92 & - & - & - \\
\hline 119 & Taraxacum officinale Wigg. & - & - & 2.03 & 0.84 & 0.46 & 133 & Withania somnifera (L.) Dunal & - & - & - & - & 0.74 \\
\hline
\end{tabular}

KEY:

LC-Lower Chail, UC-Upper Chail, S-Shinku, D-Dabargai, B-Bishigram

PDQ-Pinus-Diospyros-Quercus community, ICJ-Indigofera-Cotoneaster-Jasminium community, ACH-Apluda-Cynodon-Heteropogon community, PQJ-Pinus-Quercus-Juglans community, RIR-Rubus-Isodon-Ricinus community, AHP-Apluda-Heteropogan-Poa community, QAP-Quercus-Abies-Pinus community, SDB-Sarcococca-Daphne-Berberis community, CAB-Cynodon-Apluda-Bergenia community, CAP-Cedrus-Abies-Pinus community, PVB-Parrotiopsis-Vibernum-Berberis community, CTC-Chrysopogon-ThemedaCynodon community, PPA-Picea-Pinus-Abies community, PCI-Parrotiopsis-Cotoneaster-Indigofera community, CMR-Cenchrus-Medicago-Rumex community 


\section{Lower Chail (Site-I)}

Plant communities at this site were established at Southern and South-Eastern aspects at an elevation of $1390 \mathrm{~m}$ up to $1480 \mathrm{~m}$. Soil at this locality was loamy-sand, with $9.2 \%$ clay particles, $53.2 \%$ silt and $37.6 \%$ sand particles. Nitrogen content of the soil was estimated to be $0.1035 \%$, phosphorous $470 \mu \mathrm{g} / \mathrm{g}$, potassium $6398 \mu \mathrm{g} / \mathrm{g}$, calcium $34448 \mu \mathrm{g} / \mathrm{g}$ and magnesium $22188 \mu \mathrm{g} / \mathrm{g}$. sodium absorption ratio (SAR) of the soil was $0.52 \mathrm{millieq} / \mathrm{L}$ while sulphates were found to be $516 \mu \mathrm{g} / \mathrm{g}$. Soil $\mathrm{pH}$ at this monitoring site was recorded as 5.2 which reflected the podzolic nature of the soil. Organic matter was found to be $2.07 \%$ which is typical to the podzolic soils. $\mathrm{CaCO}_{3}$ content was low at this site i.e. $2.5 \%$ while electrical conductivity was $0.43 \mathrm{dsm}^{-1}$ (Table 3).

Table 3. Edaphic variables, mineral content and plant communities at 5 monitoring sites

\begin{tabular}{|c|c|c|c|c|c|c|c|c|}
\hline & Parameters & Units & $\begin{array}{c}\text { PDQ } \\
\text { ICJ } \\
\text { ACH }\end{array}$ & $\begin{array}{l}\text { PQJ } \\
\text { RIR } \\
\text { AHP }\end{array}$ & $\begin{array}{l}\text { QAP } \\
\text { SDB } \\
\text { CAB }\end{array}$ & $\begin{array}{l}\text { CAP } \\
\text { PVB } \\
\text { CTC }\end{array}$ & $\begin{array}{c}\text { PPA } \\
\text { PCI } \\
\text { CMR }\end{array}$ & Mean \\
\hline & & & LC & $\mathbf{U C}$ & $\mathbf{S}$ & D & $\mathbf{B}$ & \\
\hline \multirow[t]{3}{*}{1} & $\begin{array}{c}\text { Soil texture } \\
\text { Clay }\end{array}$ & $\%$ age & 9.2 & 5.2 & 7.2 & 7.2 & 9.2 & 7.6 \\
\hline & Silt & $\%$ age & 53.2 & 47.2 & 65.2 & 49.2 & 37.2 & 50.4 \\
\hline & Sand & $\%$ age & 37.6 & 47.6 & 27.6 & 43.6 & 53.6 & 42 \\
\hline 2 & $\mathrm{pH}$ & - & 5.2 & 5.4 & 5.4 & 5.8 & 6 & 5.56 \\
\hline 3 & $\mathrm{OM}$ & $\%$ age & 2.07 & 2.76 & 1.38 & 2.08 & 2.48 & 2.154 \\
\hline 4 & $\mathrm{CaCO}_{3}$ & $\%$ age & 2.5 & 7.5 & 3 & 2.5 & 10 & 5.1 \\
\hline 5 & $\mathrm{EC}$ & $\mathrm{dsm}^{-1}$ & 0.43 & 0.08 & 0.026 & 0.03 & 0.023 & 0.1178 \\
\hline 6 & Sulphates & $\mu \mathrm{g} / \mathrm{g}$ & 516 & 556 & 450 & 496 & 661 & 535.8 \\
\hline 7 & SAR & millieq/L & 0.52 & 0.2 & 0.3 & 0.13 & 0.21 & 0.272 \\
\hline 8 & $\mathrm{~N}_{2}$ & $\%$ age & 0.1035 & 0.138 & 0.069 & 0.1104 & 0.1242 & 0.10902 \\
\hline 9 & $\mathrm{P}$ & $\mu \mathrm{g} / \mathrm{g}$ & 470 & 430 & 494 & 385 & 475 & 450.8 \\
\hline 10 & $\mathrm{~K}$ & $\mu \mathrm{g} / \mathrm{g}$ & 6398 & 10634 & 8925 & 4162 & 13059 & 8635.6 \\
\hline 11 & $\mathrm{Ca}$ & $\mu \mathrm{g} / \mathrm{g}$ & 34448 & 16722 & 24148 & 39159 & 14568 & 25809 \\
\hline 12 & $\mathrm{Mg}$ & $\mu \mathrm{g} / \mathrm{g}$ & 22188 & 15433 & 17056 & 25127 & 13738 & 18708.4 \\
\hline
\end{tabular}

Key: PDQ-Pinus-Diospyros-Quercus community, ICJ-Indigofera-Cotoneaster-Jasminium community, ACH-Apluda-Cynodon-Heteropogon community, PQJ-Pinus-Quercus-Juglans community, RIRRubus-Isodon-Ricinus community, AHP-Apluda-Heteropogan-Poa community, QAP-Quercus-AbiesPinus community, SDB-Sarcococca-Daphne-Berberis community, CAB-Cynodon-Apluda-Bergenia community, CAP-Cedrus-Abies-Pinus community, PVB-Parrotiopsis-Vibernum-Berberis community, CTC-Chrysopogon-Themeda-Cynodon community, PPA-Picea-Pinus-Abies community, PCIParrotiopsis-Cotoneaster-Indigofera community, CMR-Cenchrus-Medicago-Rumex community, LCLower Chail, UC-Upper Chail, S-Shinku, D-Dabargai, B-Bishigram, OM-Organic matter, ECElectrical conductivity, SAR-Sodium Absorption Ratio, N $\mathbf{N}_{2}$-Nitrogen, P-Phosphorous, K-Potassium, Ca-Calcium, Mg-Magnesium

\section{Pinus-Diospyros-Quercus community (PDQ)}

This plant community was recorded from South facing slope at an elevation of $1480 \mathrm{~m}$. Pinus wallichiana was the dominant species with IV of 25.39. Second dominant was found to be Diospyros lotus with an IV of 23.38 , forming a close 
association with Pinus wallichiana at lower parts of the slopes. As the plant yield edible fruits with medicinal value the population density of Diospyros lotus was high with a considerably uniform dispersion. Quercus dilatata with an IV of 15.62, was mostly dispersed among Pinus wallichiana members at higher altitudes. Other prominent members of this community included Juglans rigia (12.93 IV), Ailanthus altissima (9.21 IV), Ficus carica (8.94 IV) and Crataegus songarica (4.49 IV). Common herbs growing around this community included Rumex hastatus, Solidago virga-aurea, Fragaria vesica, Plantago himalayica, Medicago polymorpha, Sonchus asper, Cuscuta europaea and Salvia moorcroftiana.

\section{Indigofera-Cotoneaster-Jasminum community (ICJ)}

On South facing slope this shrubby community was recorded. Dominant species was Indigofera heterantha var. gerardiana with an IV of 13.36 followed by Cotoneaster nummularia with an IV of 13.25. These shrubs made thick randomly dispersed patches on mostly tree-less aspect of the cliffs. Co-dominant was found to be Jasminum humile with an IV of 10.23. The rest of the shrubs included Spiraea canescens (10.01 IV), Buddleja crispa (9.6 IV), Myrsine africana (7.34 IV), Rubus fruiticosus (7.23 IV) and Daphne mucronata (6.84 IV). Other members of the shrub layer in this locality were Rubus ellipticus, Elaeagnus umbellate, Vibernum cotinifolium and Sageretia thea.

\section{Apluda-Cynodon-Heteropogon community (ACH)}

This community was recorded from South-eastern aspect at an elevation of $1390 \mathrm{~m}$. In Lower Chail area therophytes dominated the landscape. This community was dominated by Apluda mutica with an IV of 14.57, almost uniformly distributed in the locality making strong association with the second dominant Cynodon dactylon with an IV of 13.35. Just around the shrubby vegetation Apluda mutica formed mixed patches with third dominant Heteropogon contortis with an IV of 6.64. The other associated species were Fragaria vesca (5.82 IV), Bergenia ciliata (5.07 IV), Artemisia vulgaris (4.93 IV) and Origanum vulgare (4.3 IV). Remaining species such as Arthraxon prionodes, Bistorta amplexicaulis, Buplerum falcatum, Cichorium intybus, Clematis grata, Clinopodium umbrosum, Cuscuta europaea, Euphorbia prostrata, Medicago polymorpha, Pennistum orientale, Plantago himalaica, Rumex hastatus and Salvia moorcroftiana had IV values ranging between (1.32 to 3.92) (Table 2).

\section{Upper Chail (Site-II)}

Plant communities established at this monitoring site were found on an elevation of $1620 \mathrm{~m}$. Soil analysis displayed a contrasting picture with reference to adjoining Lower Chail area. Soil was silt-loam at this site with $5.2 \%$ clay particles, $47.2 \%$ silt and $47.6 \%$ sand particles. Soil reaction was mildly acidic with a soil $\mathrm{pH}$ of 5.4. Organic matter content was estimated as $2.76 \%$. Nitrogen content was $0.138 \%$ followed by phosphorous $430 \mu \mathrm{g} / \mathrm{g}$, potassium $10634 \mu \mathrm{g} / \mathrm{g}$, calcium $16722 \mu \mathrm{g} / \mathrm{g}$ and magnesium $15433 \mu \mathrm{g} / \mathrm{g}$. Sodium absorption ratio of the soil was estimated as $0.2 \mathrm{millieq} / \mathrm{L}$ and sulphate content was recorded as $556 \mu \mathrm{g} / \mathrm{g}$. Floods of recent past have eroded soil in the Upper Chail to a huge extent and main water tributary of the valley, Chail stream, is still a big threat to remaining vegetation cover in the valley floor. Highest value for $\mathrm{CaCO}_{3}$ were recorded from upper Chail i.e. $7.5 \%$ while electrical conductivity was $0.08 \mathrm{dsm}^{-1}$ (Table 3). 


\section{Pinus-Quercus-Juglans community (PQJ)}

This community was recorded at an elevation of $1620 \mathrm{~m}$, Pinus wallichiana was found to be dominated with an IV of 18.06. This was associated with Quercus baloot having an IV of 15.52. Juglans regia with an IV of 13.36 was next in abundance. These were followed by Olea ferruginea (8.42 IV), Salix denticulata (6.66 IV), Alianthus altissima (6.42) and Punica granatum (5.91 IV). In addition to this Ficus carica, Populus alba, Prunus persica, Morus nigra, Malus pumiula, Crataegus songarica and Diospyros lotus had IV values ranging between 4.32 to 2.87. Phalaris minor, Achillea millefolium, Cannabis sativa, Viola canescens, Fumaria indica, Urtica dioca, Capsella bursa-pastoris, Chenopodium album and Plantago major were the common herbaceous flora intermingled with trees association.

\section{Rubus-Isodon-Ricinus community (RIR)}

Rubus ulmifolius with an IV of 15.92 dominated this community with uniform distribution. It had thick patches of Isodon rugosus as second dominant with an IV of 13.67. But on low elevation Ricinus communis formed randomly dispersed clumps with an IV of 11.67. These were followed by Mrsine Africana (8.9 IV), Sarcococca saligna (8 IV), Indigofera heterantha var. gerardiana (7.31 IV), Jasminum officinale (6.94 IV) and Debergeasia salicifolia (6.21 IV). Other shrubby members of the community such as Daphne mucronata, Justicia adhatoda, Lespedeza juncea, Berberis lyceum and Lonicera asperifolia had IV ranging between 5.49 and 3.62.

\section{Apluda-Heteropogon-Poa community (AHP)}

This community of Upper Chail was dominated by Apluda mutica with an IV of 9.81. Though herbs were not forming a uniform cover, due to severe soil erosion by Chail stream in past few years, but still thick patches of herbs were recorded on banks of Chail stream. Second dominant species was Heteropogon contortis with an IV of 8.34 which formed association with Poa апnиa having an IV of 7.92. These dominants were followed by Phalaris minor (6.54 IV), Artemisia scoparia (5.19 IV), Urtica dioca (3.81 IV), Verbascum thapsus (3.71 IV), Plantago major (2.89 IV) and Chenopodium album (2.83 IV) (Table 2).

\section{Shinku (Site-III)}

Plant communities at this site were established at elevation of $2150 \mathrm{~m}$ on Northern and North-Western slopes. Soil analyses indicated that soils at this site are silt-loam with $7.2 \%$ clay particles, $65.2 \%$ silt particles and $27.6 \%$ sand particles. Soil pH was recorded as 5.4 and organic matter was estimated as $1.38 \%$. Nitrogen content was low i.e. $0.069 \%$. Phosphorous was estimated as $494 \mu \mathrm{g} / \mathrm{g}$ while potassium was found to be $8925 \mu \mathrm{g} / \mathrm{g}$. Calcium content was a bit higher i.e. $24148 \mu \mathrm{g} / \mathrm{g}$ and magnesium was 17056 $\mu \mathrm{g} / \mathrm{g}$ in the soils. Sodium absorption ratio was also a little low in comparison with Lower and Upper Chail i.e. 0.3 millieq/L. Sulphates were estimated as $450 \mu \mathrm{g} / \mathrm{g}$. Soil had only $3 \%$ lime and electrical conductivity remained at $0.026 \mathrm{dsm}^{-1}$ (Table 3).

\section{Quercus-Abies-Pinus community (QAP)}

Quercus-Abies-Pinus community of trees was recorded at an elevation of $1495 \mathrm{~m}$ up to $1680 \mathrm{~m}$ on Northern and North-Western slopes in Shinku area. In this community the 
dominant member was Quercus incana with an IV of 18.91. Second dominant was Abies pindrow with an IV of 18.6, making strong association with Quercus incana on North-Western aspect and Pinus wallichiana with IV of 12.56. The other important contributors to this community were Juglans regia (11.59 IV), Taxus fauna (8.88 IV), Pyrus pashia (4.16 IV) and Cedrus deodara (4.02 IV). Taxus fauna was more abundant on North-Western aspect than on Northern aspect. On lower elevations, mixed patches of Diospyros kaki (3.64 IV), Crataegus songarica (2.04 IV) and Ailanthus altissima (1.89 IV) were recorded. Some notable herbaceous members found within this community were Oxalis corniculata, Euphorbia prostrata, Anagallis arvensis, Scandix pecten-veneris, Avena fatua, Taraxacum officinale, Stellaria media, Silene conoidea, Hypericum perforatum, Poa annua and Clematis grata.

\section{Sarcococca-Daphne-Berberis community $(S D B)$}

Shrubby layer was much prominent making thick strips from Northern to NorthWestern aspect. This community was found at an elevation of $1491 \mathrm{~m}$ up to $1516 \mathrm{~m}$. Sarcococca saligna with an IV of 19.01 was the dominant making thick uniformly dispersed clumps with next dominant Daphne mucronata with an IV of 17.29. Berberis lyceum with an IV of 13.6, was common to Northern slopes. Other important members of this community included Indigifera heterantha var. heterantha (10.24 IV), Cotoneaster nummularia (7.3 IV), Myrsine africana (6.62 IV) and Buddleja crispa (6.05 IV). Rosa webbiana was randomly distributed at higher elevations with an IV of 3.51 .

\section{Cynodon-Apluda-Bergenia community $(C A B)$}

Herbaceous community was found at elevation of 1490 to $1610 \mathrm{~m}$. Cynodon dactylon appeared to be dominant with an IV of 13.97, followed by Apluda mutica with an IV of 12.47 as co-dominant. Bergenia ciliata appeared as the next dominant with an IV of 6.88 near small water falls on both aspects. This community also included Fragaria vesca (6.74 IV), Artemisia vulgaris (6.46 IV), Rumex hastatus (4.63 IV), Poa annua (4.22 IV), Sassurea albescense (4.11 IV), Avena fatua (3.53 IV) and Nacrissus tazetta (3.16 IV) (Table 2).

\section{Dabargai (Site-IV)}

Plant communities at this site were established at an elevation of $2371 \mathrm{~m}$ on NorthEastern and Southern aspects. Soil was loam with $7.2 \%$ clay particles, $49.2 \%$ silt particles and $43.6 \%$ sand particles. Soil $\mathrm{pH}$ was recorded as 5.8 and it was evident from a higher degree of podzolization in the soils of the study area with considerably low organic content i.e. $2.08 \%$. Nitrogen content was estimated as $0.1104 \%$. Phosphorous and potassium were recorded as $385 \mu \mathrm{g} / \mathrm{g}$ and $4162 \mu \mathrm{g} / \mathrm{g}$ respectively. Calcium content was found to be $39159 \mu \mathrm{g} / \mathrm{g}$ while magnesium content was $25127 \mu \mathrm{g} / \mathrm{g}$. Sodium absorption ratio was 0.13 millieq/L and sulphates were only $496 \mu \mathrm{g} / \mathrm{g} . \mathrm{CaCO}_{3}$ was estimated to be $2.5 \%$ and electrical conductivity was $0.03 \mathrm{dsm}^{-1}$ (Table 3 ).

\section{Cedrus-Abies-Pinus community (CAP)}

This tree community was found between elevation ranges of 1935 to $12371 \mathrm{~m}$. Cedrus deodara with an IV of 22.07 was the abundantly growing tree. Abies pindrow 
and Pinus wallichiana with an IV of 16.01 and 13.38 respectively were the second and third dominants. In addition to these dominant forms other important members of this community included Olea ferruginea (5.53 IV), Ficus carica (4.41 IV), Taxus fauna (4.06 IV), Quercus baloot (3.88 IV), Juglans regia (3.27 IV), Moras alba (3.02 IV) and Cedrela serrata (2.91 IV). Notable herbaceous elements of this community were Thymus linearis, Ammi visnaga, Apluda mutica, Dactylis glomerata, Centaurea iberica, Notholirion thomsonianum, Dioscorea deltoidea, Amaranthus viridis, Verbascum thapsus, Gagea elegans, Micromeria biflora and Sisymbrium irio.

\section{Parrotiopsis-Vibernum-Berberis community (PVB)}

Shrubby layer was randomly dispersed on North-Eastern and Southern slopes. Parrotiopsis jacquemontiana was dominant with an IV of 25.51 making randomly dispersed thick patches mostly on North-Eastern aspect. Vibernum grandiflorum was second dominant with an IV of 13.08 making continuous strips with Berberis lyceum (IV 12.26). Some other important contributors to this community included Indigifera heterantha var. gerardiana (7.08 IV), Maytenus royleanus (4.63 IV), Myrsine Africana (4.28 IV), Ribes himalense (4.05 IV), Isodon rugosus (3.86 IV) and Jasminum humile (3.46 IV).

\section{Chrysopogon-Themeda-Cynodon community (CTC)}

This community is dominated by Chrysopogon fulvus with an IV of 10.89 . Themeda anathera with an IV of 9.33 was more common to Southern aspect on lower elevations. Cynodon dactylon with an IV to 8.64 was the next dominant. Some other important members of this community included Avenia fetua (3.58 IV), Bergenia ciliata and Verbascum thapsus both with IV of 3.09, Salvia moorcroftiana (3.04 IV), Sisymbrium irio (2.46 IV), Cannabis sativa (2.39 IV) and Artemisis absinthium (2.36 IV) (Table 2).

\section{Bishigram (Site-V)}

Plant communities at this monitoring site were established at an elevation of $2698 \mathrm{~m}$ on Northern and Southern aspects. Soils in the area were loamy-sand in texture with $9.2 \%$ clay particles, $37.2 \%$ silt particles and $53.6 \%$ sand particles. Soil $\mathrm{pH}$ was 6 and organic matter content was estimated as $2.48 \%$. Nitrogen content was $0.1242 \%$ which was better than Lower Chail, Upper Chail and Shinku areas displaying a healthy rate of saprophytic activity in the soils. Phosphorous content was $475 \mu \mathrm{g} / \mathrm{g}$, potassium $13059 \mu \mathrm{g} / \mathrm{g}$ and calcium $14568 \mu \mathrm{g} / \mathrm{g}$. Magnesium was recorded as $13738 \mu \mathrm{g} / \mathrm{g}$. Sodium absorption ratio was low i.e. $0.21 \mathrm{millieq} / \mathrm{L}$ and sulphates were abundant i.e. $661 \mu \mathrm{g} / \mathrm{g}$. $\mathrm{CaCO}_{3}$ content was highest at this site i.e. $10 \%$ and electrical conductivity was recorded as $0.023 \mathrm{dsm}^{-1}$ (Table 3).

\section{Picea-Pinus-Abies community (PPA)}

This tree community was found at an elevation range of 2475 to $2698 \mathrm{~m}$ on both aspects. Picea smithiana with an IV of 22.07 followed by Pinus wallichiana (14.54 IV) were the leading trees in this community. On higher elevations Picea smithiana formed an association with Abies pindrow with an IV of 12.36. Other notable contributors were Juglans rigia (4.36 IV), Diospyros lotus (4.12 IV) and Ficus carica 
(3.6 IV). On Southern aspect there was a mixed patch of Platanus orientalis (3.6 IV), Acer cappadocicum and Pinus roxbergii (each with 3.07 IV). In addition to this Cedrus deodara made randomly dispersed clumps on Northern aspect with IV of 2.92. On higher elevations at Northern aspect Quercus dilatata and Taxus fauna were found with an IV of 2.79 each, delimiting a thin strip of Quercus semicarpifolia with an IV of 2.76. At this monitoring site the common herbs included Arenaria serpyllifolia, Brachiaria reptans, Eryngium coeruleum, Pseudognaphalium affine, Eragrostis minor, Angelica glauca, Calanthe tricarinata, Portulaca oleracea, Apluda mutica and Podophyllum emodi.

\section{Parrotopsis-Cotoneaster-Indigofera community (PCI)}

Parrotiopsis jacuemontiana with an IV of 16.11 making a very prominent stretched patch on Northern slopes and clumped pattern on Southern aspect. It was accompanied by Cotoneaster nummularia with an IV of 14.82. Indigofera heterantha var. heterantha was the companion shrub with an IV of 11.95. It was more common to Southern aspect than to Northern slopes. Cotoneaster nummularia displayed a uniformed dispersion on both slopes. Other important contributors to this community were Limonium cabulicum (4.96 IV), Rubus fruiticosus (4.84 IV), Jasminum humile (4.6 IV), Ribes orientale (4.54 IV), Spiraea canescens (4.47 IV) and Berberis lyceum (3.94 IV). Notable shrubs but with lesser IV included Isodon rugosus, Lespedeza juncea, Phlomis spectabilis, Andrachne cordifolia, Sarcococca saligna, Skimmia laureola and Himalrandia tetrasperma.

\section{Cenchrus-Medicago-Rumex community (CMR)}

Herbaceous community was found to have rich diversity with Cenchrus pennisetiformis as dominant, having an IV of 18.92 forming uniform strips on both slopes. It was found making good association with Medicago lupulina having an IV of 11.87. While on lower elevations Rumex hastatus with an IV of 8.97 was dominating both the aspects. This community also had Poa annua (3.8 IV), Cyanodon dactylon (3.4 IV), Aristida cyanantha (3.12 IV), and Sisymbrium irio (2.83 IV) (Table 2).

\section{Maturity index}

Maturity index was calculated according to Pichi-Sermolli (1948). At Lower Chail, tree community had 48.57 maturity index, shrubs had 31.66 and herbaceous community had a 28.61 maturity index. The mean value for the site was 36.28 . At Upper Chail, tree community showed a maturity index of 34.28 , followed by shrubby layer with a value of 23.07 and herb layer having 16.06. The mean value for these three communities was 24.47. In Shinku, tree community had a maturity index of 44 . Shrub community with a maturity index value of 27.27 while herb community had only 19.62. Mean value for maturity index at this site was 30.29. Maturity index for tree community at Dabargai area was found to be 28.88 followed by 16.31 value of shrub community and 12.73 value for herbaceous community. Mean value for the maturity index at this site was 19.30. At Bishigram, tree community had a maturity index of 27. At this site shrub had a maturity index of 20 followed by herb layer having a maturity index of 10.86. Mean value of maturity index at this site was 19.28 (Table 4). 
Table 4. Maturity index

\begin{tabular}{c|c|c|c|c|c}
\hline & Lower Chail & Upper Chail & Shinku & Dabargai & Bishigram \\
\hline Trees & 48.57 & 34.28 & 44 & 28.88 & 27 \\
Shrubs & 31.66 & 23.07 & 27.27 & 16.31 & 20 \\
Herbs & 28.61 & 16.06 & 19.62 & 12.73 & 10.86 \\
Mean & $\mathbf{3 6 . 2 8}$ & $\mathbf{2 4 . 4 7}$ & $\mathbf{3 0 . 2 9}$ & $\mathbf{1 9 . 3 0}$ & $\mathbf{1 9 . 2 8}$ \\
\hline
\end{tabular}

\section{Index of similarity}

\section{Tree communities}

According to Motyka's index of similarity (Motyka et al., 1950), PDQ tree community of Lower Chail was found to be $16.09 \%$ similar, to PQJ community of Upper Chail. The similarity between PDQ community and QAP community at Shinku was found to be $10.56 \%$. The analysis showed that similarity between PDQ community and that of CAP community at Dabargai area was $10.62 \%$. Lowest value of similarity was observed between PDQ community and PPA community of Bishigram, i.e. $10.54 \%$. Similarity between PQJ tree community of Upper Chail and that of QAP community at Shinku was $11.96 \%$. PQJ community was $12.61 \%$ like tree community CAP at Dabargai area. Only $11.09 \%$ similarity was observed between PQJ community and PPA community of Bishigram area. QAP tree community at Shinku area showed more similarity with CAP tree community of Dabargai area i.e. 16.42\%. QAP community was $16.04 \%$ like PPA community at Bishigram. CAP tree community at Dabargai area showed maximum similarity with PPA tree community at Bishigram i.e. $18.11 \%$ (Table 5).

Table 5. Similarity indices of tree communities

\begin{tabular}{c|c|c|c|c}
\hline PDQ & PQJ & QAP & CAP & PPA \\
\hline $\mathrm{X}$ & $\mathrm{X}$ & $\mathrm{X}$ & $\mathrm{X}$ & $\mathrm{X}$ \\
16.09 & $\mathrm{X}$ & $\mathrm{X}$ & $\mathrm{X}$ & $\mathrm{X}$ \\
10.56 & 11.96 & $\mathrm{X}$ & $\mathrm{X}$ & $\mathrm{X}$ \\
10.62 & 12.61 & 16.42 & $\mathrm{X}$ & $\mathrm{X}$ \\
10.54 & 11.09 & 16.04 & 18.11 & $\mathrm{X}$ \\
\hline
\end{tabular}

\section{Shrub communities}

Shrubby vegetation showed little similarities among communities based on Motyka's index. ICJ community at Lower Chail site was $6.71 \%$ like RIR community at Upper Chail. ICJ shrub community was found $10.45 \%$ similar to SDB shrub community at Shinku. Similarity between ICJ community and PVB community at Dabargai area was 7.07\%. The ICJ community was least similar to PCI community at Bishigram i.e. $5.31 \%$. Shrub community at Upper Chail i.e. RIR was $10.36 \%$ similar to SDB shrub community at Shinku. Same RIR community was $6.95 \%$ similar to PVB community of shrubs at Dabargai. A 5.17\% similarity was observed between RIR community and PCI shrub community at Bishigram. Comparison of shrub community at Shinku area i.e. SDB community and PVB community at Dabargai showed $11.81 \%$ similarity while between SDB community and PCI community at Bishigram 9.73\% similarity was 
observed. Shrub communities at Dabargai i.e. PVB and that of Bishigram i.e. PCI community showed maximum similarity i.e. $14.6 \%$ (Table 6).

Table 6. Similarity indices of shrubby communities

\begin{tabular}{c|c|c|c|c}
\hline ICJ & RIR & SDB & PVB & PC1 \\
\hline $\mathrm{X}$ & $\mathrm{X}$ & $\mathrm{X}$ & $\mathrm{X}$ & $\mathrm{X}$ \\
6.71 & $\mathrm{X}$ & $\mathrm{X}$ & $\mathrm{X}$ & $\mathrm{X}$ \\
10.45 & 10.36 & $\mathrm{X}$ & $\mathrm{X}$ & $\mathrm{X}$ \\
7.07 & 6.95 & 11.81 & $\mathrm{X}$ & $\mathrm{X}$ \\
5.31 & 5.17 & 9.73 & 14.6 & $\mathrm{X}$ \\
\hline
\end{tabular}

\section{Herbaceous communities}

Among herbaceous communities, $\mathrm{ACH}$ community at Lower Chail was $7.06 \%$ similar to AHP herb community at Upper Chail. The ACH community was found to be $19.64 \%$ similar to CAB community at Shinku while its similarity with CTC community at Dabargai site was $8.27 \%$. The herb community $\mathrm{ACH}$ was least similar to Bishigram herb community with a similarity index of just $3.03 \%$. The herb community of Upper Chail i.e. AHP was 4.57 same as CAB herb community at Shinku, 6.13\% similar to CTC community at Dabargai and 5.87\% similar to CMR community at Bishigram. The CAB herb community of Shinku was $10.68 \%$ like CTC community at Dabargai and $6.40 \%$ similar to CMR community at Bishigram. Similarty between CTC community at Dabargai and CMR community at Bishigram was found to be $6.88 \%$ (Table 7).

Table 7. Similarity indices of herbaceous communities

\begin{tabular}{c|c|c|c|c}
\hline $\mathbf{A C H}$ & AHP & CAB & CTC & CMR \\
\hline $\mathrm{X}$ & $\mathrm{X}$ & $\mathrm{X}$ & $\mathrm{X}$ & $\mathrm{X}$ \\
7.06 & $\mathrm{X}$ & $\mathrm{X}$ & $\mathrm{X}$ & $\mathrm{X}$ \\
19.64 & 4.57 & $\mathrm{X}$ & $\mathrm{X}$ & $\mathrm{X}$ \\
8.27 & 6.13 & 10.68 & $\mathrm{X}$ & $\mathrm{X}$ \\
3.03 & 5.87 & 6.40 & 6.88 & $\mathrm{X}$ \\
\hline
\end{tabular}

\section{Discussion}

Vegetation was classified into tree, shrub and herbaceous communities which is in line with the works of Ahmad et al. (2016), Dad (2016), Ali et al. (2015), Saurav and Das (2014), Sharma et al. (2014) Saglam (2013), Ahmad et al. (2010), Digiovinazzo et al. (2010), Hussain et al. (2010), Mohler et al. (2006) Munhoz et al. (2006) and Padalia et al. (2004). At Site-I, PDQ-ICJ-ACH communities were established for trees, shrubs and herbs respectively. Distribution of trees showed clumping rather than a uniform pattern of distribution due to lavish wood cutting in the area. Scanty trees and comparatively thick shrub cover near the foothills indicated the disturbed nature of habitat which is under tremendous biotic stress. Increasing population and construction of residential units is the main reason behind the species loss in this locality. Similar communities are reported from their respective study areas by Ahmad et al. (2016), Urooj et al. (2016), Haq et al. (2015), Ilyas et al. (2015), Dad (2016), Shaheen and 
Qureshi (2011), Ahmad et al. (2010), Ahmad (2009), Hussain et al. (2010) and Jabeen and Ahmad (2009). Site-II had PQJ-RIR-AHP communities. Tree and shrub members showed a clumped dispersion pattern at this monitoring site. Impacts of soil erosion were more visible at Site-I and Site-II where eroded soils had herbaceous members like Rumex hastatus, Plantago himalayica, P. major, Salvia moorcroftian and Verbascum thapsus which are indicator species of eroded soils. Similar communities but with different IV are reported by Ahmad et al. (2016), Dad (2016), Khan et al. (2016), Akhtar and Bergmeier (2015), Shaheen et al. (2015), Ilyas et al. (2015), Sharma et al. (2014), Hussain et al. (2010) and Khan et al. (2010). Site-III exhibited QAP-SDB-CAB communities. This site had a uniformly spread thick Oak forest mixed with conifers. Deforestation was also not that alarming as compared to that of Lower and Upper Chail monitoring sites. At higher elevations the forest was almost undisturbed however the habitat will face biotic stress, especially the anthropogenic pressure from increasing population in the low laying villages in next few years. Apluda mutica was observed to form randomly dispersed thick patches all along the Northern slopes at this monitoring site. These findings are backed by works of Bokhari et al. (2016), Dad (2016), Ali et al. (2015), Ilyas et al. (2015), Shaheen et al. (2015), Siddiqui et al. (2015), Ahmad et al. (2010) and Ahmad (2009). CAP-PVB-CTC communities were found at site-IV. CedrusAbies forest was much thicker on North-Eastern aspect and showed degradation on Southern slope. Chrysopogon fulvus had a uniform dispersion on North-Eastern aspect than on Southern slopes. This is line with findings of Ahmad et al. (2016), Dad (2016) Bokhari et al. (2016), Khan et al. (2016), Ali et al. (2015), Akhtar and Bergmeier (2015), Ilyas et al. (2015), Shaheen et al. (2015), Sharma et al. (2014) and Ahmad et al. (2010), Hussain et al. (2010), Ahmad (2009) Jabeen and Ahmad (2009). At site-V, PPA-PCI-CMR communities were recorded. Forest at this site was found in good health with the exception on Northern slopes where deforestation was quite evident due to fuel wood extraction by locals. These results are in line with the works of Ahmad et al. (2016), Bokhari et al. (2016), Khan et al. (2016), Akhtar and Bergmeier (2015), Haq et al. (2015), Sharma et al. (2014), Khan et al. (2012), Ahmad et al. (2010) and Hussain et al. (2010) and Ahmad et al. (2006).

\section{Conclusion}

This study reveals results of vegetation mapping based on 150 sampling units at 5 monitoring sites. Results of soil analysis cover 12 parameters. Spatial variation among plant communities at different monitoring sites is attributed to variations in edaphic variables, temperature, moisture and slope. Vegetation in the study area is threatened and needs conservation before it is too late. Anthropogenic influences, over grazing, natural calamities and erosion are reducing the vegetation cover. Expansion in agricultural fields is also causing a damage to forest cover in addition to overgrazing. Extraction of wood from forest, especially during harsh winters by the local communities, is not only reducing the tree density but is also increasing the rate of soil erosion. Climate change has not spared Hindukush Range vegetation and floral biodiversity is reducing with every passing year. Coniferous forests in this part of the world are threatened habitats because of accelerated deforestation. Impacts of anthropogenic pressure are evident from low scores for maturity index. Highest maturity index value was recorded for tree community at Site-I i.e. 48.57 followed by tree community at Site-III i.e. 44. Highest maturity index for shrub layer was recorded 
at Site-I i.e. 31.66 followed by Site-III with a value of 27.27. Similar trend was recorded for herbaceous communities. Low values for maturity index indicate inability of the plant communities to cope with anthropogenic, edaphic and climatic stresses. Similarly, low values for similarity index shows the heterogeneity among plant communities. Reforestation programs need to be initiated in the area to protect and conserve the indigenous floristic wealth. But extreme care in this regard is suggested so that no alien plant species is introduced as it will further increase stress on plants in the locality. Well trained Ecologists should be invited to chart out a plan for conservation of biodiversity in the study area. Vegetation structure is suggestive that local flora is fighting a war of its survival. Once covered beneath a thick vegetation blanket now bare patches are visible on mountain slopes. Open canopy has enabled some shrub species to make well developed layers. Provision of alternative sources of energy to the locals will reduce pressure on populations of Pinus roxburghii, P. wallichiana, Cedrus deodara, Picea smithiana, Abies pindrow and other tree species. This will in turn reduce the extant of deforestation in the locality. This study strongly recommends detailed studies on fuelwood consumption in these monitoring sites to quantify the extent of fuelwood consumption per-annum. Proper grazing management should be ensured for regeneration of understory plants. Influential elders of the area can play a vital part in protection of climax communities as well as in enforcing moderate and rotational grazing routines. Soil in the study area had low organic matter content. Acidic soils show a slow rate of humification which is the reason behind low organic matter in the soil.

\section{REFERENCES}

[1] Ahmad, I., Hussain, M., Sajid, M., Ahmad, A., Hameed, M. (2008): Spatio-temporal effects on association of plant species in Soone valley of Pakistan. - Pak. J. Bot. 40(5): 1865-1876.

[2] Ahmad, K. S., Ahmad, M., Ahmad, F., Sadia, B. (2016): Edaphic factors as major determinants of plant distribution of temperate Himalayan grasses. - Pak. J. Bot. 48(2): 567-573.

[3] Ahmad, M., Shaukat, S. S. (2012): A Textbook of Vegetation Ecology. - Abrar Sons, Karachi, Pakistan, pp: 302-305.

[4] Ahmad, M., Husain, T., Sheikh, A. H., Hussain, S. S., Siddiqui, M. F. (2006): Phytosociology and structure of Himalayan forests from different climatic zones of Pakistan. - Pak. J. Bot. 38(2): 361-383.

[5] Ahmad, M., Shoukat, S. S., Khan, D. (2010): Status of vegetation analysis in Pakistan. Int. J. Biol. Biotech. 7(3): 147-158.

[6] Ahmad, S. S. (2009): Ordination and classification of herbaceous vegetation in Margalla Hills National Park Islamabad Pakistan. - Bio. Div. Cons. 2(2): 38-44.

[7] Ahmad, S. S., Wahid, A., Akbar, K. F. (2010): Multivariate classification and data analysis of vegetation along Motorway (M-2), Pakistan. - Pak. J. Bot. 42(2): 1173-1185.

[8] Akhtar, N., Bergmeier, E. (2015): Species richness, alpha and beta diversity of trees, shrubs and herbaceous plants in the woodlands of Swat, Pakistan. - Pak. J. Bot. 47(6): 2107-2113.

[9] Ali, S., Perveen, A., Qaiser, M. (2015): Vegetation structure, edaphology and ethnobotany of Mahaban and Malka (District Buner) KPK, Pakistan. - Pak. J. Bot. 47(SI): $15-22$.

[10] Altay, V., Ozyigit, I. I., Yarc, C. (2012): Plant Communities in urban habitats of Istanbul, Turkey. - Pak. J. Bot. 44(SI): 177-186. 
[11] Angassa, A. (2005): The ecological impact of bush encroachment on the yield of grasses in Borana rangeland ecosystem. - Afri. J. Eco. 43(1): 14-20.

[12] Badshah, L., Hussain, F., and Sher, Z. (2016): Floristic inventory, ecological characteristics and biological spectrum of plants of Parachinar, Kurram Agency, Pakistan. - Pak. J. Bot. 48(4): 1547-1558.

[13] Bokhari, T. Z., Liu, Y., Ahmad, M., Siddiqui, M. F., Li, Q., Khan, Z., Malik, S. A. (2016): Multivariate analysis of some Pine forested areas of Azad Kashmir, Pakistan. Pak. J. Bot. 48(1): 105-113.

[14] Bouyoucos, G. J. (1936): Directions for making mechanical analysis of soils by the hydrometer method. - Soil Sci. 42: 225-228.

[15] Brady, N. C. (1990): The Nature and Properties of Soils. 10th ed. - Macmillan Publishing Co., New York, NY.

[16] Bremner, J. M., Mulvaney, C. S. (1982): Nitrogen - Total. - In: Page, A. L. et al. (eds.) Methods of Soil Analysis. Agronomy Monograph 9, Part 2, 2nd ed. American Society of Agronomy, Madison, WI, pp. 595-624.

[17] Dad, J. M. (2016): Distribution, species diversity and composition of plant communities in relation to various affecting factors in an Alpine grassland at Bandipora, Kashmir. Pak. J. Bot. 48(2): 551-560.

[18] Digiovinazzo, P., Ficetola, G. F., Bottoni, L., Andreis, C., Schioppa, E. P. (2010): Ecological thresholds in herb communities for the management of suburban fragmented forests. - Forest Ecology \& Management 259(3): 343-349.

[19] Dondeyne, S., Wijffels, A., Emmanuel, L. B., Deckers, J., Hermy, M. (2004): Soils and vegetation of Angai forest: ecological insights from a participatory survey in South Eastern Tanzania. - Afri. J. Eco. 42(3): 198-207.

[20] Gould, W. A., Gonzalez, G., Carrero, R. G. (2006): Structure and composition of vegetation along an elevational gradient in Puerto Rico. - J. Vegetation Science 17: 563574.

[21] Grillini, C. R. L., Orioli, L., Piussi, P., Kakuru, W. (2001): Structural analysis of the Chambura Gorge forest (Queen Elizabeth National Park, Uganda). - Afr. J. Ecology 38(4): 295-302.

[22] Haq, F., Ahmad, H., Iqbal, Z. (2015): Vegetation description and phytoclimatic gradients of subtropical forests of Nandiar Khuwar catchment District Battagram. - Pak. J. Bot. 47(4): 1399-1405.

[23] Hussain, A., Farooq, M. A., Ahmad, M., Akbar, M., and Zafar, M. U. (2010): Phytosociology and structure of central Karakoram national park (CKNP) of Northern areas of Pakistan. - World App. Sci. J. 9(12): 1443-1449.

[24] Hussain, A., Mirza, S. N., Khan, I. A., Naeem, M. A. (2009): Determination of relative species composition and seasonal plant communities of Nurpur reserved forest in scrub rangelands of District Chakwal. - Pak. J. Agri. Sci. 46(1): 55-59.

[25] Hussain, F. (1989): Field and Laboratory Manual of Plant Ecology. - UGC, Islamabad.

[26] Hussain, F., Badshah, L. (1998): Vegetation structure of Pirghar Hills South Waziristan, Pakistan. - J. Tropical \& Subtropical Bot. 6: 187-195.

[27] Hussain, F., Illahi, I. 1(991): Ecology and Vegetation of Lesser Himalayan Pakistan. Bot. Dept., Univ. of. Peshawar, Peshawar.

[28] Hussain, F., Iqbal, I., Akhtar, P. (2005): Floristic and vegetation studies of Ghalegay Hills, District Swat, Pakistan. - Inter. J. Biol. \& Biotech. 2(4): 847-852.

[29] Ilyas, M., Qureshi, R., Akhtar, N., Munir, M., Ziaulhaq. (2015): Vegetation analysis of Kabal valley, District Swat, Pakistan using multivariate approach. - Pak. J. Bot. 47(SI): 77-86.

[30] Jabeen, T., Ahmad, S. S. (2009): Multivariate analysis of environmental and vegetation data of Ayub National Park, Rawalpindi. - Soil \& Env. 28(2): 106-112.

[31] Jackson, M. L. (1962): Soil Chemical Analysis. - Constable \& Co., Ltd., London, p. 406407. 
[32] Khan, A., Ahmad, M., Siddiqui, M. F., Iqbal, J., Wahab, M. (2016): Phytosociological analysis of Pine forest at Indus Kohistan, KPK, Pakistan. - Pak. J. Bot. 48(2): 575-580.

[33] Khan, N., Ahmad, M., Wahab, M., Nazim, K., Ajaib, M. (2010): Phytosociology, structure and physicochemical analysis of soil in Quercus baloot Griff. Forest District Chitral, Pakistan. - Pak. J. Bot. 42(4): 2429-2441.

[34] Khan, N., Ahmed, M., Siddiqui, M. F., Bibi, S., Ahmed, I. (2012): A phytosociological study of forest and non-forest vegetation of District Chitral, Hindukush Range, Pakistan. - FUUAST J. Bio. 2(1): 91-101.

[35] Lovett, J. C., Marshall, A. R., Carr, J. (2006): Changes in tropical forest vegetation along an altitudinal gradient in the Udzungwa Mountains National Park, Tanzania. - Afr. J. Ecology 44(4): 478-490.

[36] Luis, C., Eurico, M., Adelia, D. M. (2002): Vegetation structure and ecology of the Cufada Lagoon (Guinea-Bissau). - Afr. J. Eco. 40(3): 252-259.

[37] Maestre, F. T., Bradford, M. A., Reynolds, J. F. (2006): Soil heterogeneity and community composition jointly influence grassland biomass. - J. Vegetation. Sci. 17(3): 261-270.

[38] Mishra, N. K., Singh, R., Ojha, S., Supreeti. (2012): Phytosociological perspectives of representative herbaceous Genera of common occurrence belonging to family Asteraceae in Grassland ecosystem of Anpara region in District Sonebhadra (U.P.). - Ind. J. L. Sci. 2(1): 119-122.

[39] Mohler, C. L., Marks, P. L., Gardescu, S. (2006): Guide to the Plant Communities of Central Finger Lakes Region. - New York State Agricultural Experiment Station, New York, pp. 7-9.

[40] Motyka, J., Dobrzanski, B., Zawadski, S. (1950): Wstepne badania nad lakami polundnlowowschodneij Lubeiszczyzny. - Ann. Univ. M. Curie-Sklodowska 13: 367447.

[41] Munhoz, C. B. R., Felfili, J. M. (2006): Phytosociology of the herb-subshrub layer in an area of Campo Sujo, Distrito Federal, Brazil. - Acta Bot. Bras. 20(3). http://dx.doi.org/10.1590/S0102-33062006000300017.

[42] Nagendra, H., Utkarsch, G. (1993): Landscape ecological planning through a multi-scale characterization of pattern: studies in Western Ghats, South India. - Environ. Monit. Assess. 87(3): 215-233.

[43] Noor, A., Khatoon, S. (2013): Analysis of vegetation pattern and soil characteristics of Astore Valley Gilgit Baltistan. - Pak. J. Bot. 45(5): 1663-1667.

[44] Padalia, H., Chauhan, N., Porwal, M. C., Roy, P. S. (2004): Phytosociological observations on tree species diversity of Andaman Islands, India. - Current Sci. 87: 799806.

[45] Pichi-Sermolli, R. E. (1948): An index for establishing the degree of maturity in plant communities. - J. Ecology 36: 85-90.

[46] Rayan, J. N., Harvey, R. W., Metge, D. W., Larson, J. E. (1997): Transport of bacteriophage PRD1 and silica colloids in a sewage-contaminated aquifer. - EOS, Transactions of the American Geophysical Union 86, F231. Presented at the Fall Meeting of the American Geophysical Union.

[47] Rhoades, J. D. (1996): Salinity: Electrical Conductivity and Total Dissolved Solids. - In: Sparks, D. L. (ed.) Methods of Soil Analysis: Chemical Methods, Part 3. ASA and SSSA, Madison, WI, pp. 417-435.

[48] Richard, L. A. (1954): Diagnoses and Improvement of Saline and Alkali Soils. Agriculture Hand Book. - USDA, Washington DC, USA.

[49] Saglam, C. (2013): A phytosociological study of the forest, shrub, and steppe vegetation of Kizildag and environs (Isparta, Turkey). - Turk. J. Bot. 37: 316-335.

[50] Salvatori, V., Egunyu, F., Skidmore, A. K., Leeuw, J. D., Van-Gils, H. A. M. (2003): The effects of fire and grazing pressure on vegetation cover and small mammal populations in the Maasai Mara National Reserve. - African J. Ecology 39(2): 200-204. 
[51] Sarmah, K., Jha, L. K., Tiwari, B. K. (2011): Spatio-temporal changes in land uses and land cover due to human interference in Meghalaya, India: A case study of Wah Shella micro-watershed. - Journal of Geography and Regional Planning 4(6): 338-343.

[52] Saurav, M., Das, A. P. (2014): Plant species richness and phytosociological attributes of the vegetation in the cold temperate zone of Darjilling Himalaya, India. - Int. Res. J. Env. Sci. 3(10): 47-57.

[53] Shah, M., Hussain, F. (2009): Phytosociological studies of the vegetation of Hayat Abad Peshawar, Pakistan. - Pak. J. Pl. Sci. 15(2): 123-128.

[54] Shaheen, H., Qureshi, R. A. (2011): Vegetation types of Sheosar lake and surrounding landscape in Deosai plains of North Pakistan, Western Himalayas. - J. Med. Pl. Res. 5(4): 599-603.

[55] Shaheen, H., Sarwar, R., Firdous, S. S., Dar, M. E. I., Zahidullah., Khan, S. M. (2015): Distribution and structure of conifers with special emphasis on Taxus baccata in moist temperate forests of Kashmir Himalayas. - Pak. J. Bot. 47(SI): 71-76.

[56] Sharma, P., Rana, J. C., Devi, U., Randhawa, S. S., Kumar, R. (2014): Floristic diversity and distribution pattern of plant communities along altitudinal gradient in Sangla valley, Northwest Himalaya. - The Sci. World. J. http://dx.doi.org/10.1155/2014/264878.

[57] Siddiqui, M. F., Ahmed, M., Shaukat, S. S., Khan, N. (2010): Advance multivariate techniques to investigate vegetation-environmental complex of pine forests of moist area of Pakistan. - Pak. J. Bot. 42: 267-293.

[58] Siddiqui, M. F., Ahmed, M., Hussain, S. S., Shaukat, S. S., Khan, N. (2011): Vegetation description and current status of moist temperate coniferous forests of Himalayan and Hindukush region of Pakistan. - FUUAST J. Biol. 1(2): 99-114.

[59] Siddiqui, M. F., Salam, A., Ahmad, M., Hussain, M. I., Iqbal, J., Wahab, M. (2015): Present state and future trends of Pine forests of Malam Jabba, Swat District, Pakistan. Pak. J. Bot. 47(6): 2161-2169.

[60] Sorensen, T. (1948): A method of establishing groups of equal amplitudes in plant sociology based on similarity of species content and its application to analyze the vegetation of Darnish commons. - Biol. Skr. 5: 1-34.

[61] Urooj, R., Ahmad, S. S., Ahmad, M. N., Ahmad, H., Nawaz, M. (2016): Ordination study of vegetation analysis around wetland area: A case study of Mangla Dam, Azad Kashmir, Pakistan. - Pak. J. Bot. 48(1): 115-119.

[62] Wahab, M., Ahmad, M., Khan, N. (2008): Phytosociology and dynamics of some pine forests of Afghanistan. - Pak. J. Bot. 40(3): 1071-1079.

[63] Wahab, M., Ahmad, M., Khan, N., Sarangzai, A. M. (2010): A phytosociological study of pine forest from District Dir, Pakistan. - J. Biol. Biotech. 7(3): 219-226.

[64] Wazir, S. M., Dasti, A. A., Saima, S., Shah, J., Hussain, F. (2008): Multivariate analysis of vegetation of Chapursan Valley: an alpine meadow in Pakistan. - Pak. J. Bot. 40(2): 615-626. 